\title{
REDES DE COLABORAÇÃO CIENTÍFICA NA ÁREA DE QUÍMICA NO BRASIL: UM ESTUDO BASEADO NAS COAUTORIAS DOS ARTIGOS DA REVISTA QUÍMICA NOVA
}

Cristina Gomes de Souza*, Rafael Garcia Barbastefano e Leonardo Silva de Lima

Departamento de Engenharia de Produção, Centro Federal de Educação Tecnológica Celso Suckow da Fonseca, Av. Maracanã, 229, Bl. E, 20271-110 Rio de Janeiro - RJ, Brasil

Recebido em 18/3/11; aceito em 25/10/11; publicado na web em 4/1/12

\begin{abstract}
CHEMISTRY COLABORATION NETWORKS IN BRAZIL: A COAUTHORSHIP STUDY IN QUIMICA NOVA ARTICLES. In this paper we built three co-authorship networks displaying the acquaintances between countries, universities and authors that have published papers in Quimica Nova from 1995 to 2008. Our research was conducted applying a bibliometric approach to 1782 papers and over 4200 authors. Centrality measures were used and the most significant actors of each network were pointed out. The results using the centrality metrics and the network structures indicated that Quimica Nova resembles a typical scientific community.
\end{abstract}

Keywords: social network analysis; co-authorship ; centrality measures.

\section{INTRODUÇÃO}

A dinâmica existente entre pesquisadores e instituições formando redes de interação que envolvem a troca de informações, o compartilhamento de recursos e a difusão do conhecimento configura-se, ao mesmo tempo, como resultado e força motriz do desenvolvimento científico e tecnológico em determinado campo do saber. A identificação dos atores que compõem essas redes e da natureza e intensidade do vínculo que os une, permite o mapeamento dos principais atores e dos agrupamentos formados.

Existem medidas topológicas aplicadas a redes que são capazes de identificar os agrupamentos já estabelecidos, bem como o surgimento de novos grupos de pesquisa através da chamada Análise de Redes Sociais (ARS). A ARS consiste no estudo de uma estrutura formada por um conjunto finito de atores e das relações existentes entre eles. ${ }^{1}$ A definição dos atores de uma rede permite flexibilidade e depende do problema que se pretende investigar. No caso de colaboração científica, por exemplo, esse ator pode ser um pesquisador (nível micro), uma instituição (nível meso), um país (nível macro). ${ }^{2}$ A partir dos laços relacionais verificados entre os atores pode-se construir um modelo de representação da organização dessa rede de colaboração apoiando-se em conceitos e métricas oriundos da teoria dos grafos.

O estudo de redes de colaboração científica tem suscitado grande interesse no meio acadêmico, observando-se a realização de pesquisas - publicadas em veículos de reconhecido mérito internacional ${ }^{3}$ - voltadas para o desenvolvimento de metodologias e análises que buscam melhor compreensão desse fenômeno social que se verifica em todas as áreas do conhecimento, com maior intensidade nas ciências naturais, ${ }^{4}$ embora venha ocorrendo um aumento progressivo na área das ciências sociais. ${ }^{5}$

Uma manifestação da colaboração científica se dá através da relação de coautoria, em que pesquisadores dividem a responsabilidade e o mérito pela publicação dos resultados de pesquisas desenvolvidas em parceria. ${ }^{4} \mathrm{~A}$ prática de escrever artigos em coautoria tem se intensificado nas últimas décadas, sendo que esse aumento ocorre tanto em incidência (fração de artigos que apresentam coautoria) quanto em extensão (quantidade de coautores nas publicações). ${ }^{6}$

$\mathrm{O}$ aumento da realização de trabalhos em coautoria pode ser explicado em função das novas Tecnologias da Informação e Comuni-

\footnotetext{
*e-mail: cgsouza@cefet-rj.br
}

cação (TICs), que favorecem o trabalho colaborativo à distância; ${ }^{6}$ das políticas governamentais e das agências de fomento que estimulam a cooperação interinstitucional e internacional; ${ }^{7}$ dos altos custos de $\mathrm{P} \& \mathrm{D}$, que fazem com que pesquisadores compartilhem recursos e infraestrutura; da necessidade de especialização, principalmente nas áreas em que a instrumentalização é complexa, fazendo com que a colaboração ocorra em função da necessidade de divisão do trabalho e, da interdisciplinaridade da ciência que demanda pesquisadores advindos de diferentes áreas de conhecimento. ${ }^{7}$

Em 2007, a Sociedade Brasileira de Química, SBQ, comemorou 30 anos. A revista Química Nova, na ocasião, editou um número especial, em que foi publicado o artigo A Importância das Revistas Química Nova e Journal of the Brazilian Chemical Society no Crescimento da Área de Química no Brasil mostrando o panorama atual, a evolução e os desafios desses dois periódicos. ${ }^{8}$ Trata-se de duas publicações editadas pela SBQ, que contam com o reconhecimento da comunidade científica, sendo que a Química Nova é considerada um dos marcos históricos da entidade.

Dada a importância desse periódico, o presente trabalho teve por objetivo mostrar como se estrutura a rede de colaboração científica entre os autores da Química Nova, a partir de relações de coautoria dos artigos publicados desde a sua indexação na base do ISI/Web of Science. Adotando-se a premissa de que a Química Nova é representativa dos esforços de pesquisas realizados pela comunidade de química no Brasil e que as relações de coautoria retratam a colaboração científica nesta área, espera-se dar uma contribuição para melhor compreensão da organização social dessa área de conhecimento no país.

$\mathrm{O}$ trabalho encontra-se organizado em seções. A seção 2 aborda as redes sociais e explica as propriedades analisadas em relação à rede de coautoria da Química Nova. A seção 3 descreve o método utilizado. A seção 4 apresenta e discute os resultados encontrados. Por fim seguem as considerações finais.

\section{REDES SOCIAIS E MÉTRICAS DE CENTRALIDADE}

Os estudos de redes sociais datam dos anos 30, sendo que sua origem advém do campo das ciências sociais e do comportamento. Foi recentemente, entretanto, que seu uso se propagou, levando ao desenvolvimento e sofisticação de modelos e métodos quantitativos voltados para análise da estrutura dessas redes sociais. ${ }^{9}$

$\mathrm{Na}$ ARS o foco do estudo não está nos atributos dos atores que 
compõem a rede, mas nas relações que os unem. Através da ARS é possível identificar aspectos, tais como, padrões de relacionamento entre os atores de uma rede; a conectividade entre os mesmos; a formação de clusters; a evolução da rede ao longo do tempo e, o fluxo de comunicação, informação e conhecimento dentro da rede. Em se tratando de rede de coautoria de artigos científicos, podem ser estabelecidos padrões de relação entre os pesquisadores responsáveis pela produção do conhecimento, o papel desempenhado por cada pesquisador dentro da rede e outras informações de interesse para a compreensão da dinâmica da organização da pesquisa numa área específica do saber.

Uma rede social pode ser representada por um grafo $G=(V, E)$ formado por Vértices $(V)$ e Arestas $(E)$. Cada vértice representa um ator e cada aresta representa a relação existente entre dois atores integrantes da rede. No caso de uma rede de coautoria, os vértices representam os autores, suas instituições ou países de origem e as arestas representam os artigos produzidos em parceria. As arestas também podem ser valoradas. No caso da rede de coautoria, essa valoração se dá em função do número de artigos publicados em conjunto.

A importância relativa de cada ator dentro da rede pode ser identificada através de métricas de centralidade. No escopo desse estudo foram utilizadas as seguintes medidas de centralidade: de grau; de proximidade (closeness) e, de intermediação (betweenness). Além das medidas de centralidade, outra variável interessante a ser observada numa rede de coautoria é a chamada componente gigante. A seguir são apresentadas as métricas de centralidade e a definição de componente gigante.

\section{Centralidade de grau}

O grau de um vértice $v$ corresponde ao número de arestas incidentes ou ao número de vértices adjacentes a ele. Em uma rede de coautoria esse grau vai indicar o total de atores da rede que publicaram em parceria com um determinado ator. O grau de centralidade poderá variar, portanto, de 0 (publicação sem parceria com outros atores) até $n-1$ (publicação com todos os demais atores da rede excluindo a si próprio), onde $n$ é o número total de atores da rede.

Embora o grau de centralidade de um vértice indique o número de conexões de um ator, trata-se de uma medida de caráter eminentemente local. Em uma determinada rede vários atores podem possuir o mesmo grau de centralidade mas, ao se observar a posição relativa dos mesmos, alguns podem deter uma centralidade global mais evidente que outros. Assim é interessante que a centralidade de grau de um vértice seja avaliada juntamente com outras medidas de centralidade.

\section{Centralidade de proximidade}

A centralidade de proximidade é definida pela soma das distâncias geodésicas entre um determinado vértice e todos os outros vértices do grafo (distância geodésica é o menor caminho - número de arestas - que liga dois vértices de uma rede). Essa medida indica a proximidade de um determinado ator em relação aos demais atores da rede. Matematicamente, a centralidade de proximidade pode ser definida como: ${ }^{10}$

$$
C_{C}(v)=\frac{n-1}{\sum_{t \in V / v} d_{G}(v, t)}
$$

onde $d_{G}(v, t)$ corresponde à distância geodésica entre os vértices $v$ e $t$.

De acordo com a expressão acima, na rede de coautoria, um ator com uma centralidade de proximidade alta pode indicar que tenha uma maior possibilidade de estabelecer mais parcerias de publicação na rede por estar mais próximo em relação a todos os outros atores.

\section{Centralidade de intermediação}

A centralidade de intermediação atribui importância a um ator em função do fluxo que passa por ele para interligar outros dois atores da rede, através do menor caminho possível. Essa medida foi definida por Freeman como: ${ }^{11}$

$$
\operatorname{Bet}(v)=\sum_{i \neq j \neq v, i \neq v} \frac{\sigma_{i, j}(v)}{\sigma_{i, j}}
$$

onde $\sigma_{\mathrm{i}, \mathrm{j}}$ é o número de caminhos geodésicos que ligam os vértices $i$ e $j$; enquanto $\sigma_{\mathrm{i}, \mathrm{j}}(v)$ representa o número de caminhos geodésicos que ligam os vértices $i$ e $j$ passando por $v$. Se um vértice $v$ tem alto valor de centralidade de intermediação, então $v$ ocorre um maior número de vezes entre os menores caminhos entre todos os pares de vértices do grafo que os demais vértices $u \in V$.

$\mathrm{Na}$ rede de coautoria, um vértice com alto valor de centralidade de intermediação indica que um número significativo das parcerias estabelecidas na rede envolve, de forma direta ou indireta, as publicações relacionadas a esse ator.

\section{Componente gigante}

A componente gigante consiste no maior subgrafo conexo de uma rede, ou seja, maior subconjunto formado por vértices e arestas em que há, pelo menos, um caminho entre quaisquer pares de vértices. No caso da rede de coautoria, essa componente gigante irá indicar o percentual de autores que, direta ou indiretamente, possui ligação com os demais integrantes da rede.

\section{PARTE EXPERIMENTAL}

A pesquisa foi realizada na base Web of Science do ISI Web of Knowledge, acessada através do Portal de Periódicos da CAPES, utilizando como termo de busca 'Química Nova' no campo nome da publicação. A consulta, realizada em 2009, recuperou 2.515 documentos abrangendo desde o volume 18 , número 3 , publicado em 1995, até o volume 31, número 6, que veio a ser a última publicação de 2008. Quanto ao tipo de documento recuperado, obtiveram-se 1.782 artigos científicos (70,9\% dos registros obtidos). O ISI cataloga outros documentos como Editoriais, Revisões e Cartas de Leitores que não foram incluídos nas análises.

O processo de obtenção dos grafos envolveu primeiramente a determinação dos atores (vértices) das redes sociais. Em cada registro de produção dos artigos, foram obtidos os autores, as instituições e os países envolvidos. Os nomes passaram por um processo de depuração para evitar a geração de vértices múltiplos representando o mesmo ator (por exemplo, em um artigo a instituição pode ser registrada como "USP”, em outro como "Universidade de São Paulo"). Importante destacar que, como toda base de dados, a recuperação da informação é feita conforme sua indexação. Isso implica a possibilidade de haver percentual de perdas de informação provenientes de erros de digitação e de preenchimento incorreto, incompleto ou não uniforme (principalmente nos campos autor e instituição). Vale mencionar ainda que a indexação é feita a partir dos dados constantes no documento quando de sua publicação, independente de mudanças que possam acontecer ao longo do tempo como, por exemplo, de vínculo institucional do autor. Essa possível perda de informação, entretanto, não é estatisticamente considerada significativa conforme estudo de Newman. ${ }^{12}$

As relações entre os vértices foram estabelecidas através da coautoria, ou seja, caso dois pesquisadores sejam autores de um mesmo artigo, então os vértices que os representam serão ligados por uma aresta na rede dos autores. As instituições dos autores, bem como 
seus países, também foram ligadas nas respectivas redes. As arestas nas três redes foram valoradas pelo número de artigos verificados em coautoria.

Para cada uma das redes, foram calculadas as métricas de centralidade (grau, proximidade e intermediação) para todos os vértices envolvidos.

\section{RESULTADOS E DISCUSSÃO}

Ao longo do período de tempo abrangido pelo estudo, pode-se observar a evolução da quantidade de artigos, de países, de instituições e de autores que tiveram trabalhos publicados no periódico Química Nova. A Tabela 1 mostra o quantitativo de 1995 (a partir do vol. 18, n. 3) e, desde então, o total acumulado até 2000, 2005 e 2007.

Tabela 1. Quantitativo acumulado de artigos, países, instituições e autores de artigos publicados em Química Nova

\begin{tabular}{lcccc}
\hline & Até $12 / 95$ & Até $12 / 00$ & Até $12 / 05$ & Até $12 / 08$ \\
\hline Artigos & 62 & 560 & 1.178 & 1.782 \\
Países & 9 & 25 & 30 & 30 \\
Instituições & 34 & 155 & 289 & 423 \\
Autores & 145 & 1.256 & 2.611 & 4.281 \\
\hline
\end{tabular}

Com base nos dados da Tabela 1, observa-se que, ao longo do período analisado, os 1.782 artigos publicados na Química Nova tiveram 4.281 autores, vinculados a 423 instituições oriundas de 30 países diferentes. Os dados da Tabela 1 mostram ainda a evolução do número de países, instituições e autores ao longo do tempo, ou seja, a evolução do número de nós (atores) que compõem a rede de coautoria da Química Nova considerando-se o nível macro (países), meso (instituições) e micro (autores). Importante destacar que dos 1.782 artigos, apenas 182 (10,2\% do total) foram escritos por um autor apenas. Os demais 1.600 artigos $(89,8 \%)$ envolveram relações de coautoria.

\section{Rede de países}

Conforme mostrado na Tabela 1 , foi verificado vínculo dos autores com 30 países. A distribuição dos artigos pelos países, entretanto, é bastante desigual, havendo forte concentração no Brasil. Do total de 1.782 artigos, quase $95 \%$ deles apresentam autores vinculados a instituições brasileiras. Na sequência aparece vínculo com Portugal, França e Espanha. A Tabela 2 apresenta o quantitativo de artigos dos países que tiveram, no mínimo, 10 publicações em QN.

Como o foco do trabalho consiste nas relações de colaboração, é importante a identificação da quantidade de artigos publicados em parceria. Conforme observado na Tabela 3, 115 artigos (menos de $10 \%$ do total) foram desenvolvidos em parceria por autores de diferentes países sendo que, dentre esses, 108 artigos apresentaram colaboração entre dois países.

O grafo que representa a relação de colaboração entre países é mostrado na Figura 1. Conforme pode ser observado através da espessura das arestas que ligam dois países, as relações mais intensas de colaboração acontecem principalmente entre Brasil e Alemanha e entre Brasil e Espanha. Na sequência, as relações são entre Brasil e Inglaterra e entre Brasil e Portugal. Com menor intensidade, mostra relações do Brasil com USA, Canadá, Argentina, Itália e Cuba.

A partir dos resultados apresentados podem ser feitas as seguintes considerações: quando há colaboração do Brasil com outros países, esta ocorre principalmente com países europeus, tanto em quantidade quanto em intensidade; a colaboração com EUA e Canadá, embora
Tabela 2. Relação de países com, pelo menos, 10 artigos publicados em Química Nova no período analisado

\begin{tabular}{ccc}
\hline País & Artigos & $\%$ \\
\hline Brasil & 1597 & $94,6 \%$ \\
Portugal & 29 & $1,7 \%$ \\
França & 23 & $1,4 \%$ \\
Espanha & 22 & $1,3 \%$ \\
Argentina & 19 & $1,1 \%$ \\
Alemanha & 16 & $0,9 \%$ \\
Chile & 15 & $0,9 \%$ \\
Inglaterra & 15 & $0,9 \%$ \\
USA & 13 & $0,8 \%$ \\
Colômbia & 10 & $0,6 \%$ \\
Canadá & 10 & $0,6 \%$ \\
\hline
\end{tabular}

Tabela 3. Distribuição do número de países em cada artigo

\begin{tabular}{ccc}
\hline Países por artigo & Artigos & $\%$ \\
\hline 1 & 1667 & 93,5 \\
2 & 108 & 6,1 \\
3 & 6 & 0,3 \\
6 & 1 & 0,1 \\
Total & 1782 & 100,0 \\
\hline
\end{tabular}

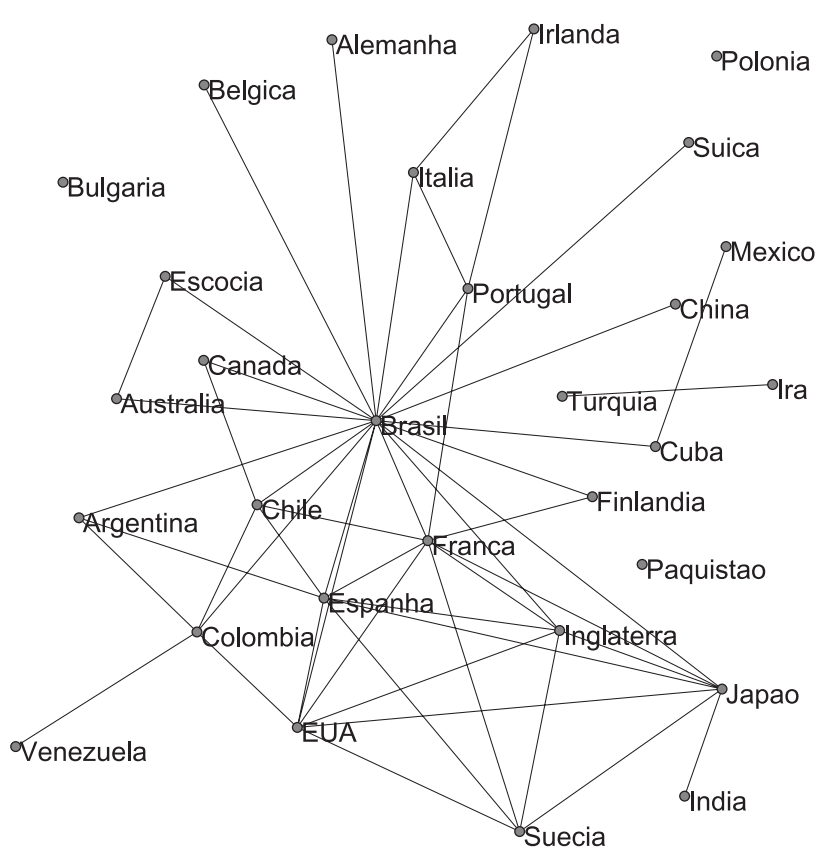

Figura 1. Rede de países

menos intensa do que com Espanha, Alemanha, Portugal e Inglaterra, é mais representativa do que com outros países das Américas; a colaboração do Brasil com países da América do Sul é tênue, tendo ocorrido apenas com Argentina, Chile e Colômbia e, mesmo assim, em quantidade reduzida de artigos; é muito pequena a colaboração com países asiáticos ou com outras economias emergentes, verificando-se poucas publicações, apenas com Japão e China; também é pequena a quantidade de artigos envolvendo parceria internacional que não contou com a participação do Brasil, sendo que a distância máxima entre os demais países e o Brasil é de duas arestas - com 
exceção dos países que não estão conectados direta ou indiretamente no grafo. A posição central ocupada pelo Brasil na rede de colaboração entre países pode ser constatada através das métricas de centralidade relacionadas na Tabela 4, em que o país ocupa a primeira posição em todas elas (grau, intermediação e proximidade).

Tabela 4. Ranking dos principais países em relação às medidas de grau, intermediação e proximidade

\begin{tabular}{cc}
\hline País & Grau \\
\hline Brasil & 19 \\
Franca & 9 \\
Espanha & 8 \\
Japão & 7 \\
EUA & 7 \\
\hline País & Intermediação \\
\hline Brasil & 0,505659 \\
Japão & 0,061846 \\
Colômbia & 0,058908 \\
Cuba & 0,056650 \\
Franca & 0,039415 \\
\hline País & Proximidade \\
\hline Brasil & 0,689655 \\
Franca & 0,487805 \\
Espanha & 0,465116 \\
EUA & 0,465116 \\
Japão & 0,454545
\end{tabular}

\section{Rede de instituições}

Os 1.782 artigos analisados nesse estudo foram oriundos de 423 instituições, como consta na Tabela 1. A distribuição dos artigos pelas instituições também se apresenta bastante heterogênea. A Tabela 5 apresenta o quantitativo de artigos das instituições que tiveram, no mínimo, 35 publicações em Química Nova.

Tabela 5. Instituições com maior número de publicações no periódico

\begin{tabular}{|c|c|}
\hline Instituição & Artigo \\
\hline USP & 429 \\
\hline UNICAMP & 237 \\
\hline UFRJ & 184 \\
\hline UNESP & 145 \\
\hline UFSCar & 124 \\
\hline UFMG & 116 \\
\hline UFBA & 79 \\
\hline UFSC & 78 \\
\hline UFRGS & 66 \\
\hline UFPR & 57 \\
\hline UFPE & 54 \\
\hline UFV & 52 \\
\hline UFC & 46 \\
\hline UFF & 45 \\
\hline UEM & 37 \\
\hline UFRRJ & 35 \\
\hline
\end{tabular}

Entretanto, dessas 1.782 publicações, 61,2\% do total (1.090 artigos) foram escritos por autores com vínculo com uma única instituição, ou seja, sem colaboração interinstitucional (Tabela 6). Excluindo-se os 182 artigos escritos por um único autor, tem-se que 908 artigos (aproximadamente 51\% do universo analisado) envolveram relações de colaboração intrainstitucional. Das 692 publicações com coautoria interinstitucional, a grande maioria (506 artigos) foi escrita em parceria entre duas instituições. A colaboração entre 3 ou mais instituições aconteceu em 186 artigos.

Tabela 6. Distribuição do número de instituições em cada artigo

\begin{tabular}{ccc}
\hline Instituições por artigo & Artigos & $\%$ \\
\hline 1 & 1090 & 61,2 \\
2 & 506 & 28,4 \\
3 & 145 & 8,1 \\
4 & 29 & 1,6 \\
5 & 8 & 0,4 \\
7 & 2 & 0,1 \\
8 & 1 & 0,1 \\
9 & 1 & 0,1 \\
Total & 1782 & 100 \\
\hline
\end{tabular}

É importante notar que as instituições que mais publicam e também as mais centrais na rede são aquelas que possuem alguns dos programas de pós-graduação mais bem avaliados pela CAPES na área de Química (Tabela 7). Tal fato reforça a posição da revista como pólo de atração dos interesses da comunidade científica de Química.

Tabela 7. Centralidade das instituições (Avaliação CAPES - Programa melhor avaliado e Num. Progs - o número de programas de pós-graduação avaliados na área de Química na Trienal 04-06)

\begin{tabular}{lccccc}
\hline Nome & $\begin{array}{c}\text { Cent. } \\
\text { Prox. }\end{array}$ & $\begin{array}{c}\text { Cent. } \\
\text { Interm. }\end{array}$ & $\begin{array}{c}\text { Cent. } \\
\text { Grau }\end{array}$ & $\begin{array}{c}\text { Aval. } \\
\text { CAPES }\end{array}$ & $\begin{array}{c}\text { Núm. } \\
\text { Progs. }\end{array}$ \\
\hline USP & 0,409 & 0,203 & 86 & 7 & 4 \\
UNICAMP & 0,381 & 0,084 & 62 & 7 & 1 \\
UFRJ & 0,379 & 0,110 & 59 & 6 & 5 \\
UNESP & 0,378 & 0,110 & 65 & 7 & 1 \\
UFSC & 0,364 & 0,079 & 40 & 7 & 1 \\
UFSCar & 0,361 & 0,035 & 43 & 7 & 1 \\
UFMG & 0,355 & 0,077 & 46 & 6 & 1 \\
UFBA & 0,349 & 0,034 & 36 & 5 & 1 \\
EMBRAPA & 0,340 & 0,016 & 23 & - & - \\
UFRGS & 0,340 & 0,090 & 32 & 6 & 2 \\
\hline
\end{tabular}

\section{Rede de autores}

De acordo com dados da Tabela 1, 4.281 autores foram responsáveis pela elaboração dos 1.782 artigos analisados neste estudo. A distribuição do número de autores por artigo está registrada na Tabela 8, de modo a ilustrar a incidência e a extensão da colaboração científica existente no universo de publicações que fizeram parte do trabalho.

Uma característica verificada na rede de autores é o aparecimento de uma Componente Gigante (Figura 2), representando mais de $50 \%$ dos autores, o que revela que a publicação Química Nova 
Tabela 8. Autores por artigo

\begin{tabular}{ccc}
\hline Autores por artigo & Artigos & $\%$ \\
\hline 1 & 182 & 10,2 \\
2 & 400 & 22,4 \\
3 & 429 & 24,1 \\
4 & 340 & 19,1 \\
5 & 203 & 11,4 \\
6 & 108 & 6,1 \\
7 & 64 & 3,6 \\
8 & 23 & 1,3 \\
9 & 9 & 0,5 \\
10 ou mais & 24 & 1,3 \\
Total & 1782 & 100 \\
\hline
\end{tabular}

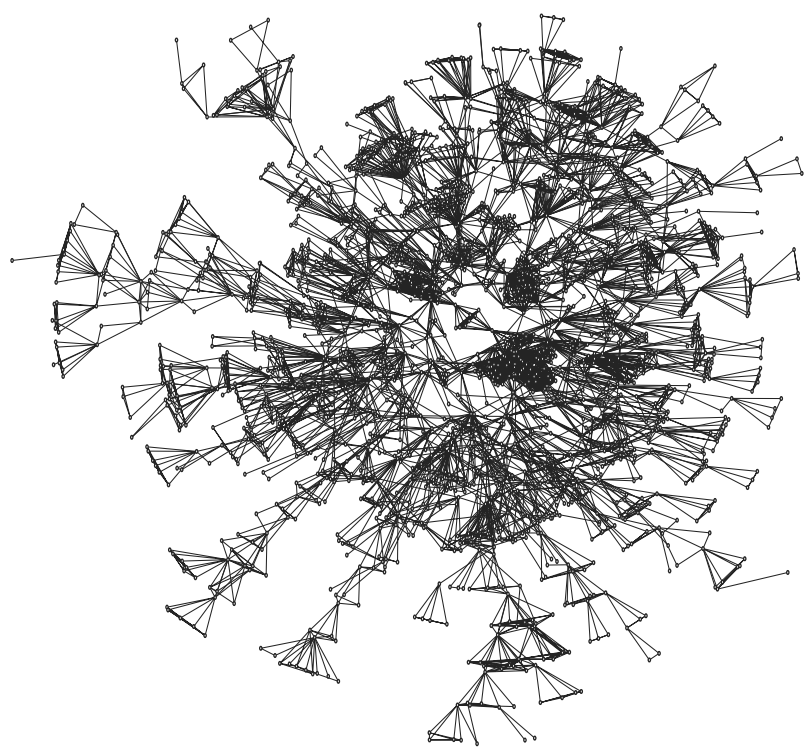

Figura 2. Componente gigante de autores

possui características típicas de uma comunidade científica, conforme apontado por Newman. ${ }^{12}$

A relação dos pesquisadores (identificados como pesquisador 1, 2, 3 etc.) com as maiores medidas de centralidade de intermediação é apresentada na Tabela 9. Foi usada esta métrica para ordenar os pesquisadores em função do seu caráter mais global e que ressalta importância maior no processo de disseminação do conhecimento em se tratando de rede de coautoria.

Conforme observado na Tabela 10, verifica-se que os autores com maior centralidade são reconhecidos pesquisadores na comunidade científica brasileira, possuindo bolsa de produtividade em pesquisa (PQ) do CNPq majoritariamente no nível 1. Tais pesquisadores também atuam em programas de pós-graduação considerados de excelência, muitos dos quais, avaliados pela Capes com conceitos $7 \mathrm{e}$ 6; contribuem para a formação de novos pesquisadores considerando o número de orientações de doutorado concluídas e, podem ser considerados seniores se tomarmos o tempo de titulação de doutorado.

\section{CONSIDERAÇÕES FINAIS}

O estudo mostrou a rede de colaboração existente entre os autores dos artigos publicados em Química Nova a partir da relação de coautoria. Tratou-se de um estudo baseado em métricas quantitativas, considerando-se que a coautoria é um indicador de colaboração científica.
Tabela 9. Centralidade dos autores

\begin{tabular}{cccc}
\hline Pesquisador & Intermediação & Proximidade & Grau \\
\hline 1 & 0,065 & 0,096 & 28 \\
2 & 0,063 & 0,098 & 54 \\
3 & 0,059 & 0,1 & 19 \\
4 & 0,044 & 0,096 & 42 \\
5 & 0,04 & 0,088 & 41 \\
6 & 0,037 & 0,087 & 32 \\
7 & 0,029 & 0,088 & 24 \\
8 & 0,028 & 0,091 & 38 \\
9 & 0,028 & 0,087 & 46 \\
10 & 0,028 & 0,086 & 14 \\
11 & 0,027 & 0,078 & 21 \\
12 & 0,025 & 0,09 & 14 \\
13 & 0,02 & 0,087 & 24 \\
14 & 0,02 & 0,078 & 17 \\
15 & 0,019 & 0,092 & 36 \\
\hline
\end{tabular}

Tabela 10. Perfil dos autores com maiores medidas de centralidade

\begin{tabular}{ccccc}
\hline Pesq. & $\begin{array}{c}\text { Bolsa } \\
\text { CNPq }\end{array}$ & $\begin{array}{c}\text { Conceito } \\
\text { programa } \\
\text { pós-graduação }\end{array}$ & $\begin{array}{c}\text { Orientaçães } \\
\text { concluídas } \\
\text { doutorado }\end{array}$ & $\begin{array}{c}\text { Anos de } \\
\text { titulação do } \\
\text { doutorado }\end{array}$ \\
\hline 1 & $1-B$ & 7 & 21 & 18 \\
2 & $1-A$ & 7 & 27 & 26 \\
3 & 2 & 7 & 8 & 20 \\
4 & $1-B$ & 6 & 15 & 29 \\
5 & $1-D$ & 6 & 12 & 21 \\
6 & $1-B$ & 7 & 20 & 19 \\
7 & $1-A$ & 7 & 26 & 18 \\
8 & 2 & $5 *$ & 5 & 32 \\
9 & SR & 5 & 25 & 40 \\
10 & $1-D$ & 6 & 12 & 20 \\
11 & 2 & 7 & 5 & 11 \\
12 & 2 & 6 & 18 & 30 \\
13 & $1-D$ & 6 & 13 & 24 \\
14 & $1-D$ & 6 & 29 & 33 \\
15 & $1-B$ & 6 & 27 & 29 \\
\hline
\end{tabular}

Para uma compreensão da organização social da pesquisa na área de Química no Brasil, seria interessante o aprofundamento do presente estudo, procurando-se investigar as motivações que levam os pesquisadores a colaborarem, o que compreendem como colaboração e coautoria e as consequências dessas colaborações.

A colaboração a nível macro (países) ainda é reduzida, abrangendo menos de $10 \%$ dos artigos publicados. Nesse nível de colaboração, o Brasil apresenta posição destacada configurando-se como ator central dentro da rede de coautoria. Esse resultado já era esperado uma vez que se trata de um periódico brasileiro altamente qualificado que se volta, principalmente, para a comunidade científica da Química no Brasil.

No nível meso de colaboração, verifica-se que instituições com programas de pós-graduação bem avaliados pela CAPES também são instituições centrais. Resultado semelhante aparece no nível micro, 
onde autores centrais também são reconhecidos na comunidade pelas Bolsas de Produtividade em Pesquisa do CNPq.

Os autores formam uma rede com componente gigante abrangendo mais de $50 \%$ do total dos vértices. Pode-se deduzir que Química Nova, mais que uma publicação, teria já características de comunidade científica real.

\section{REFERÊNCIAS}

1. Wasserman, S.; Faust, K.; Social Network Analysis: Methods and Applications, 16 ${ }^{\text {th }}$ ed., Cambridge University Press: New York, 2007.

2. Glanzel, W.; Lib. Trends 2002, 50, 461.

3. Palla, G.; Barabasi, A.; Vicsek, T.; Nature 2007, 446, 664; Barabasi, A.; Jeong, H.; Neda, Z.; Ravasz, E.; Schubert, A.; Vicsek, T.; Phys. A 2002, 311, 590; Nagpaul, P.; Scientometrics 2002, 54, 213; Jiang, Y.; Scientometrics 2008, 74, 471; Li-Chun, Y.; Kretschmer, H.; Hanneman, R.; Ze-Yuan, L.; Inf. Process. Manage. 2006, 42, 1599.
4. Balancieri, R.; Bovo, A.; Kern, V.; Pacheco, R.; Barcia, R.; C. Inf. 2005, 34, 64; Cronin, B.; Shaw, D.; la Barre, K.; J. Am. Soc. Inf. Sci. Technol. 2003, 54, 855; Acedo, F.; Barroso, C.; Casanueva, C.; Galán, J.; J. Manage. Stud. 2006, 43, 957.

5. Moody, J.; Am. Sociol. Rew. 2004, 69, 213.

6. Laband, D.; Tollison, R.; J. Pol. Econ. 2000, 108, 632.

7. Kretschmer, H.; Scientometrics 2004, 60, 409; Lee, S.; Bozeman, B.; Soc. Stud. Sci. 2005, 35, 673; Matheus, R.; Vanz, S.; Moura, A.; Anais do VII Congreso Iberoamericano de Indicadores de Ciencia y Tecnología, São Paulo, Brasil, 2007.

8. de Torresi, S. I. C.; Pardini, V. L.; Dias, L. C.; Pinto, A. C.; de Andrade, J. B.; Magalhães, M. E. A.; Gil, P. E. A.; Quim. Nova 2007, 30, 1491.

9. Wasserman, S.; Scott, J.; Carrington, P.; Models and Methods in Social Network Analysis, Cambridge University Press: New York, 2005.

10. de Nooy, W.; Mrvar, A.; Batagelj, V.; Exploratory Social Network Analysis with Pajek, Cambridge University Press: New York, 2005.

11. Freeman, L. C.; Soc. Nets. 1979, 1, 215.

12. Newman, M.; Phys. Rev. E: Stat., Nonlinear, Soft Matter Phys. 2001, 64, 016131. 